\title{
Tailored Subcycle Nonlinearities of Ultrastrong Light-Matter Coupling
}

\author{
J. Mornhinweg $\odot,{ }^{1}$ M. Halbhuber, ${ }^{1}$ C. Ciuti $\odot,{ }^{2}$ D. Bougeard, ${ }^{1}$ R. Huber $\odot,{ }^{1, *}$ and C. Lange $\odot^{1,3, \dagger}$ \\ ${ }^{1}$ Department of Physics, University of Regensburg, 93040 Regensburg, Germany \\ ${ }^{2}$ Université de Paris, laboratoire Matériaux et Phénomènes Quantiques, CNRS, F-75013 Paris, France \\ ${ }^{3}$ Fakultät Physik, Technische Universität Dortmund, 44227 Dortmund, Germany
}

(Received 8 June 2020; revised 23 September 2020; accepted 16 February 2021; published 28 April 2021)

\begin{abstract}
We explore the nonlinear response of tailor-cut light-matter hybrid states in a novel regime, where both the Rabi frequency induced by a coherent driving field and the vacuum Rabi frequency set by a cavity field are comparable to the carrier frequency of light. In this previously unexplored strong-field limit of ultrastrong coupling, subcycle pump-probe and multiwave mixing nonlinearities between different polariton states violate the normal-mode approximation while ultrastrong coupling remains intact, as confirmed by our mean-field model. We expect such custom-cut nonlinearities of hybridized elementary excitations to facilitate nonclassical light sources, quantum phase transitions, or cavity chemistry with virtual photons.
\end{abstract}

DOI: 10.1103/PhysRevLett.126.177404

Exposing solid-state electrons to extremely strong optical fields can drive highly nonperturbative dynamics faster than an oscillation cycle of the carrier wave, generating high-harmonic or high-order sideband radiation [1-4]. For a field resonant to an electronic excitation, the Rabi frequency $\Omega_{R}$ quantifies the rate of periodic absorption and stimulated emission. When $\Omega_{R}$ exceeds the carrier frequency of light $\omega_{c}$, carrier-wave Rabi flopping occurs, accompanied by strong nonlinearities $[5,6]$.

In a complementary setting, strongly enhanced vacuum field fluctuations of subwavelength microcavities can be coupled to electronic excitations, causing a periodic energy exchange between the electronic transition and the cavity field at a rate called the vacuum Rabi frequency $\Omega_{R}^{v}$. As a consequence, light-matter hybrid states-cavity polaritons - emerge, with custom optical and electronic properties. When $\Omega_{R}^{v}$ approaches or even exceeds $\omega_{c}$, ultrastrong [7-16] and deep-strong coupling [12,17] emerge, respectively. Under such exotic conditions, antiresonant interactions can simultaneously create (or annihilate) both a cavity photon and an electronic excitation. As a result, the ground state contains a sizable population of virtual photons. Even under equilibrium conditions examined by weak probe pulses, this limit of light-matter interaction has brought forth complex quantum effects including the vacuum BlochSiegert shift [13], modified electronic transport [14,18], cavity chemistry $[19,20]$, or vacuum-field-induced superconductivity $[21,22]$.

Combining both scenarios, intense laser fields are expected to drive strong-field nonlinearities of light-matter coupled hybrid states in a situation where $\Omega_{R}^{v}$ and $\Omega_{R}$ simultaneously become comparable to $\omega_{c}$, leading to subcycle polarization dynamics driven by both the vacuum field and a coherent field. Such forced interactions of normal modes would produce previously inaccessible optical and electronic states beyond the eigenstates of the light-matter coupled system, unfolding an unprecedented parameter space for exploring dynamical quantum phenomena. Moreover, nonlinearities may be exploited to tailor correlations or phase transitions, and facilitate new devices including sources of single [23] and entangled photons, or nonclassical quantum states of light [24].

Here, we explore this novel strong-field limit, by driving ultrastrongly cavity-coupled Landau electrons $\left(\Omega_{R}^{v} / \omega_{c}=0.77\right)$ by strong, single-cycle terahertz pulses reaching $\Omega_{R}=2 \pi \times 2.7 \mathrm{THz}$ [Fig. 1(a)]. With $\Omega_{R}^{v} \approx \omega_{c} \approx \Omega_{R}$, nonperturbative excitations of the hybrid system including coherent Landau ladder climbing by up to 10 rungs occur on subcycle timescales. Strong pump-probe and coherent four- [25], six-, and eight-wave-mixing nonlinearities occur for each polariton branch individually. Importantly, controlled nonlinear interactions between individual polaritons arise, dynamically breaking the normal-mode approximation. Our theory quantitatively reproduces these data and links the nonlinearities to dynamical Coulomb correlations during nonperturbative excitation of Landau electrons. This first exploration of strong-field interactions of normal modes of ultrastrong coupling paves the way for designing light-matter hybrid states with custom-cut nonlinear functionalities.

Our structures couple the eigenmodes of metallic terahertz resonators to the cyclotron resonance (CR) of two-dimensional electron gases hosted in $6 \mathrm{GaAs}$ quantum wells (QWs), $n$ doped with an electron density of $1.75 \times$ $10^{12} \mathrm{~cm}^{-2}$ [Fig. 1(a)]. A variable magnetic bias field $B$ Landau quantizes the electrons and sets the CR frequency $\nu_{c}$. Single-cycle terahertz waveforms [Fig. 1(a), red waveform] probe the transmission spectrum of the bare 
resonators and reveal a fundamental LC mode at a frequency of $\nu_{\mathrm{LC}}=\omega_{\mathrm{LC}} / 2 \pi=0.81 \mathrm{THz}$ and a dipolar mode at $\nu_{\mathrm{DP}}=1.8 \mathrm{THz}$. Including the QWs, the electric field of these modes ultrastrongly couples to the polarization of the CR. As $\nu_{c}$ is varied, the transmission spectrum reveals several minima corresponding to three pronounced light-matter coupled modes [Fig. 1(b)]. A lower polariton (LP) branch emerges from the CR at low frequencies, while a corresponding upper polariton (UP) resonance is observed at a frequency of $1.5 \mathrm{THz}$ for $\nu_{c}=0 \mathrm{THz}$. As $\nu_{c}$ is increased, the polariton frequencies grow with opposite curvatures, causing the characteristic anticrossing shape. Off-resonant coupling of the CR to the dipolar cavity mode creates a third resonance labeled DPP, located $\approx 0.2 \mathrm{THz}$ below the UP branch (Supplemental Material [26]). Terahertz absorption by the CR in uncoupled areas between resonator structures explains the transmission minimum at $\nu_{c}$. Diagonalizing the lightmatter Hamiltonian for the LP and UP resonances, we obtain a coupling strength of $\Omega_{R}^{v} / \omega_{\mathrm{LC}}=0.77$ [26].

We interrogate the system's nonlinear response by field-resolved two-dimensional spectroscopy [6,29-32]. This characterization strategy singles out and identifies all multiphoton interaction pathways separately by their Liouville paths. Two single-cycle terahertz waveforms labeled A and B with a relative time delay $\tau$ and peak amplitudes of 1.3 and $2.5 \mathrm{kV} / \mathrm{cm}$, respectively, generated by tilted-pulse front optical rectification [33], are collinearly focused through the structure [Fig. 2(a)] and detected by electro-optic sampling. Mechanical choppers enable us to measure the transmitted fields $\mathcal{E}_{\mathrm{A}}$ and $\mathcal{E}_{\mathrm{B}}$ of both pulses individually, as well as the combined field $\mathcal{E}_{\mathrm{AB}}$, from which we determine the nonlinear response, $\mathcal{E}_{\mathrm{nl}}=\mathcal{E}_{\mathrm{AB}}-\mathcal{E}_{\mathrm{A}}-\mathcal{E}_{\mathrm{B}}$.

First, we switch off the magnetic field $\left(\nu_{c}=0 \mathrm{THz}\right)$ and investigate the nonlinearities of the cavity coupled to the electron plasma, where the relevant UP and DPP modes assume frequencies of 1.5 and $1.2 \mathrm{THz}$, respectively [Fig. 1(b)]. The nonlinear response $\mathcal{E}_{\text {nl }}$ displays a rich time-domain structure with multiple oscillation patterns [Fig. 2(b)]. Fronts of constant phase of pulse A appear as vertical lines, while those of pulse B manifest as diagonal lines for which $t-\tau$ is constant. $\mathcal{E}_{\mathrm{nl}}$ is strongest around $\tau=0 \mathrm{ps}$, where both pulses interfere constructively, causing an oscillation with the frequencies of the UP and the DPP. The resulting pronounced beating along the $t$ axis periodically minimizes the envelope of $\mathcal{E}_{\mathrm{nl}}$ at $t \cong 3$ and $5.5 \mathrm{ps}$. For $\tau= \pm 0.25 \mathrm{ps}$, destructive interference of the pulses minimizes the terahertz peak amplitude and, therefore, $\mathcal{E}_{\mathrm{nl}}$.

Next, we set $B$ to $2.3 \mathrm{~T}$ and measure $\mathcal{E}_{\mathrm{nl}}$ at the anticrossing point, $\nu_{c} \approx \nu_{\mathrm{LC}}$, where the LP, UP, and DPP resonances are centered at frequencies of $0.4,1.65$, and $1.3 \mathrm{THz}$, respectively [Fig. 1(b)]. Now, a higher level of coherence is apparent by the slower decay of $\mathcal{E}_{\mathrm{nl}}$, leading to a finite amplitude for delay times up to $\tau=5$ ps [Fig. 2(c)] [26]. Moreover, here, the beating pattern is caused by interactions of three resonances. The resulting complex nature of $\mathcal{E}_{\text {nl }}$ gives reason to expect a rich structure of nonlinearities.

For a systematic decomposition of all nonlinear optical interaction processes, we perform a two-dimensional Fourier transform [6,29-32]. The resulting nonlinear amplitude
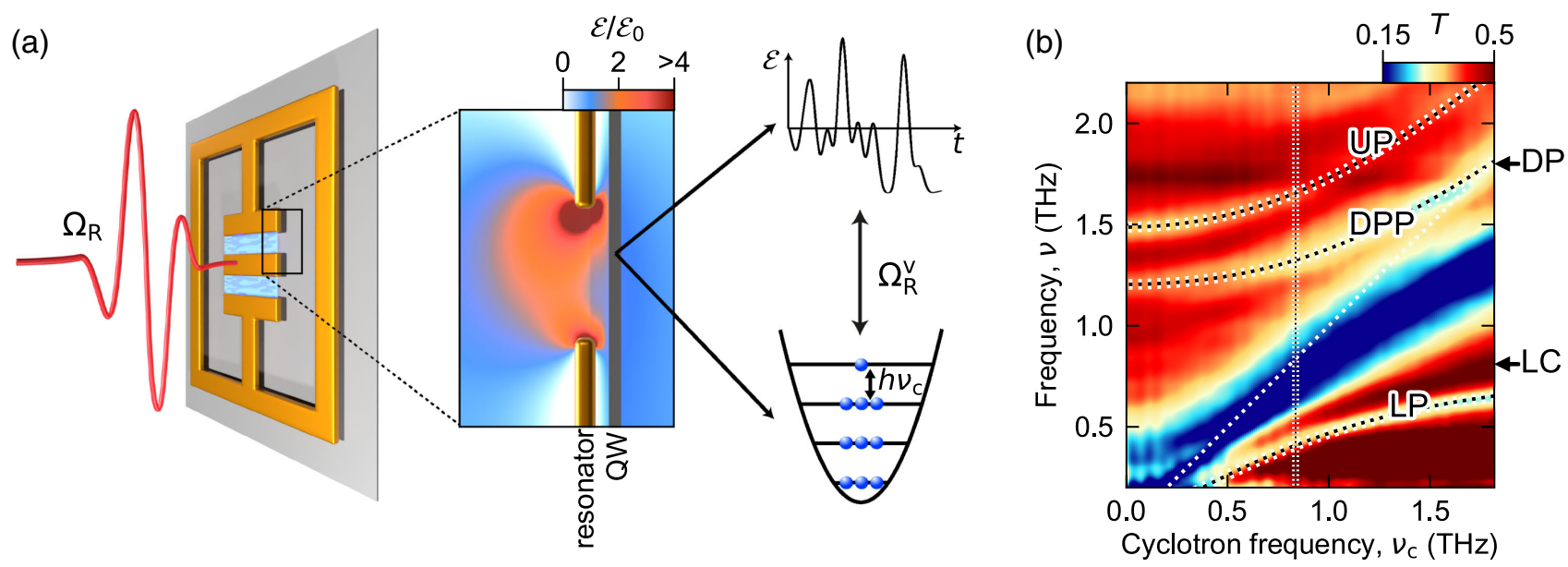

FIG. 1. Ultrastrongly light-matter coupled structure. (a) The terahertz far field (red waveform) couples to the modes of the resonator (gold structure) with a strength defined by the Rabi frequency $\Omega_{R}$. Gray layer: quantum wells (QWs). The magnified area shows the near field amplitude inside the resonator gap. Here, vacuum field fluctuations of the resonator mode (upper right-hand graph) couple to the Landau levels (lower right-hand schematic) of the QWs with a vacuum Rabi frequency $\Omega_{R}^{v}$. (b) Measured transmission spectra as a function of the cyclotron frequency $v_{c}$. The dotted curves show the lower (LP) and upper polariton mode (UP) obtained from Hopfield's model for a coupling strength of $\Omega_{R}^{v} / \omega_{c}=0.77$. DPP: additional polariton mode. The vertical dotted line marks the anticrossing point, $v_{c}=0.84 \mathrm{THz}$. The diagonal dotted line highlights the cyclotron resonance. The arrows indicate the frequencies of the uncoupled LC and DP cavity modes, respectively. 

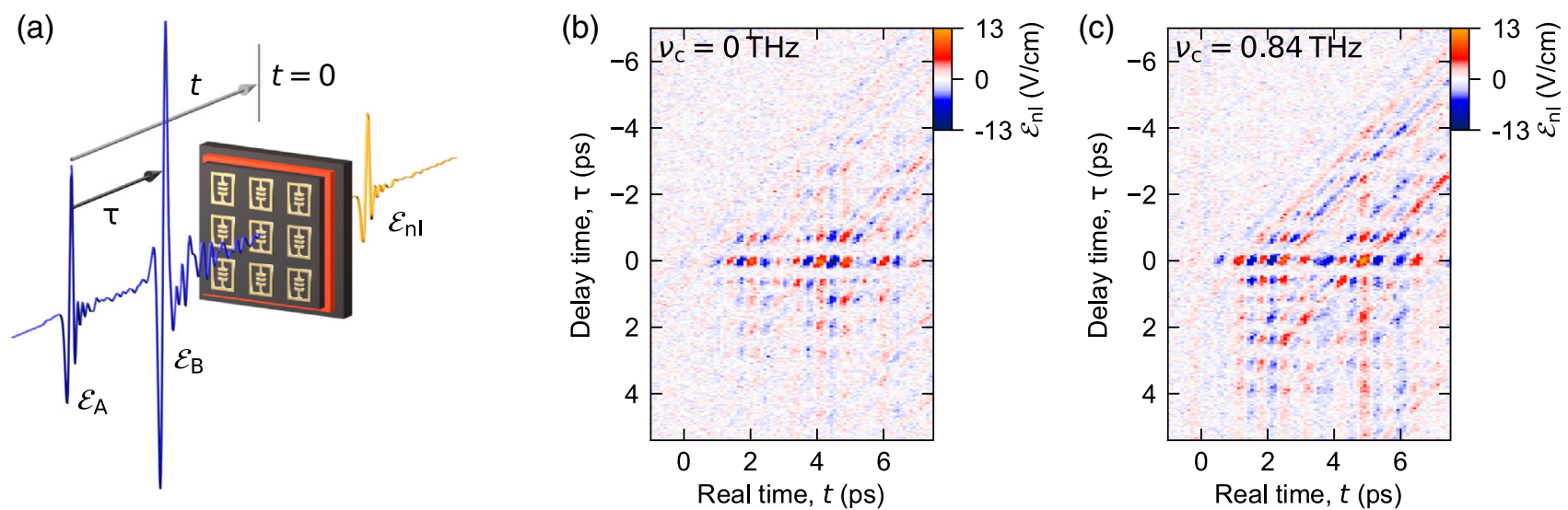

FIG. 2. (a) Experimental geometry of two-dimensional terahertz spectroscopy. $t$ is the electro-optic delay time (real time). $\tau$ is the relative delay of the two terahertz waveforms. (b) Measured nonlinear response $\mathcal{E}_{\mathrm{nl}}(t, \tau)$ for $\nu_{c}=0 \mathrm{THz}$. (c) $\mathcal{E}_{\mathrm{nl}}(t, \tau)$ at the anticrossing point, where $\nu_{c} \approx \nu_{\mathrm{LC}}$.

spectrum, $\mathcal{A}_{\mathrm{nl}}\left(\nu_{t}, \nu_{\tau}\right)$, depends on the frequencies $\nu_{t}$ and $\nu_{\tau}$ associated with the respective delay times. For $\nu_{\tau}=0 \mathrm{THz}$, $\mathcal{A}_{\mathrm{nl}}$ reveals three distinct, narrowband signatures at the polariton frequencies [Fig. 3(a)]. Generally, each maximum of $\mathcal{A}_{\mathrm{nl}}$ corresponds to a distinct multiphoton process combining the pseudo-wave vectors of the incident fields, $\mathbf{k}_{\mathrm{A}}=\left(\nu_{j}, 0\right)$ and $\mathbf{k}_{\mathrm{B}}=\left(\nu_{j}, \nu_{j}\right)$, where $\nu_{j}$ with $j \in\{\mathrm{LP}, \mathrm{UP}, \mathrm{DPP}\}$ denotes the polariton frequencies, and off-resonant spectral components can be neglected $[6$, 29-32]. Peaks along the black dashed lines mark pump- probe $(\mathrm{PP})$ processes resulting from third-order nonlinearities, $P_{\mathrm{PP}}^{(3)}\left(\nu_{j}\right)=\chi^{(3)}\left(\nu_{j}, \nu_{j},-\nu_{j}\right) \mathcal{A}_{m}\left(\nu_{j}\right) \mathcal{A}_{n}\left(\nu_{j}\right) \mathcal{A}_{n}\left(-\nu_{j}\right)$, whereby $\chi^{(3)}$ is the corresponding nonlinear susceptibility and $\mathcal{A}_{m}$ with $m, n \in\{\mathrm{A}, \mathrm{B}\}, m \neq n$, denotes the spectral amplitudes of the incident fields. The resulting wave vectors, $\mathbf{k}_{\mathrm{PP} 1}=\mathbf{k}_{\mathrm{A}}+\mathbf{k}_{\mathrm{B}}-\mathbf{k}_{\mathrm{B}}$ and $\mathbf{k}_{\mathrm{PP} 2}=\mathbf{k}_{\mathrm{B}}+\mathbf{k}_{\mathrm{A}}-\mathbf{k}_{\mathrm{A}}$, cause a cancellation of the phase of either one of the incident field components, showing that the PP processes probe the population response. In contrast, four-wave-mixing
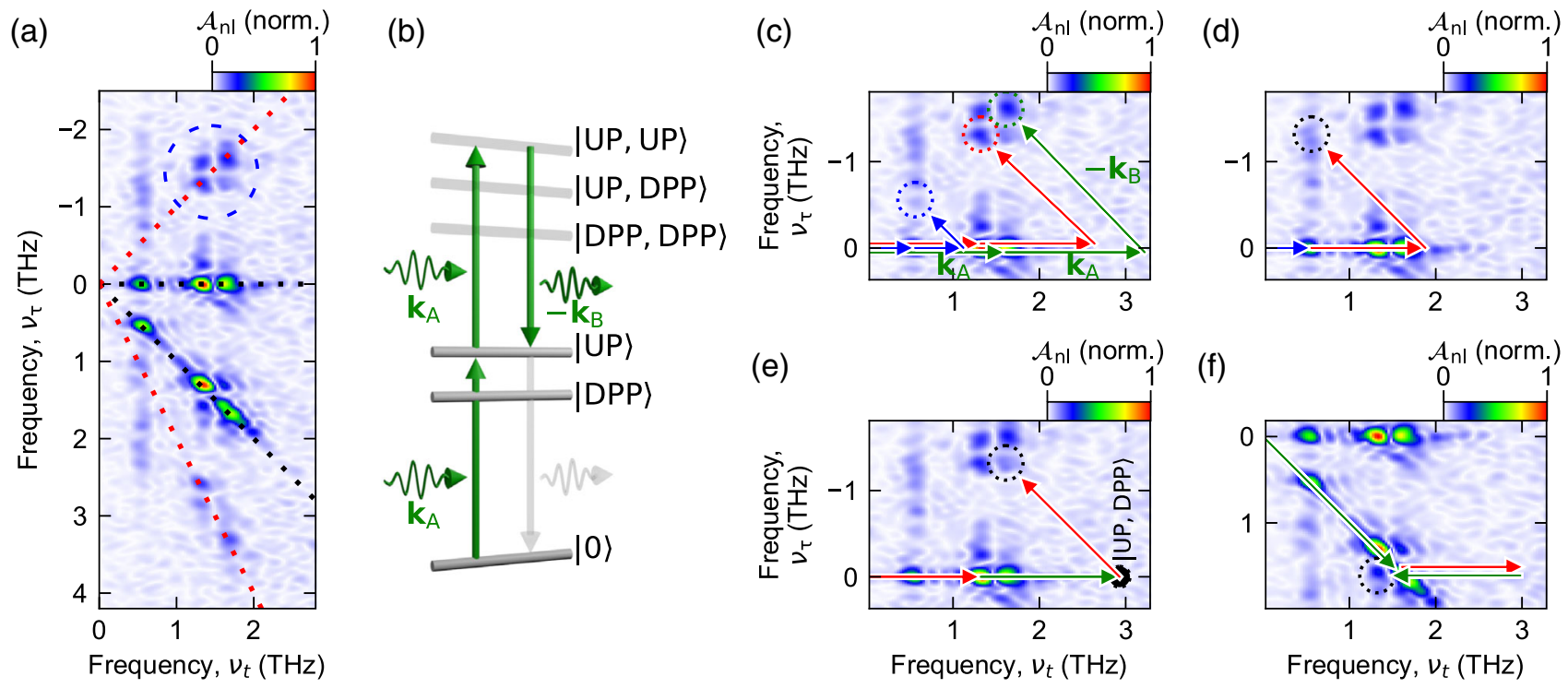

FIG. 3. (a) Experimental amplitude spectrum $\mathcal{A}_{\mathrm{nl}}\left(\nu_{t}, \nu_{\tau}\right)$ of the time-domain data of Figure 2(c), normalized to its peak value. Dashed lines as guides to the eye mark third-order processes with only one resonance. The dashed circle shows four-wave mixing processes of the UP and the DPP. (b) Schematic of a four-wave-mixing process. Two photons from field A with wave vectors $\mathbf{k}_{\mathrm{A}}$, resonant to the UP (left-hand green waveforms), create a virtual excitation which is mixed down to the polariton frequency by emission of a photon into field $\mathrm{B}$ with wave vector $\mathbf{k}_{\mathrm{B}}$ (right-hand green waveform). Reemission into the far field is illustrated by the light gray arrow. Other virtual levels $|\mathrm{UP}, \mathrm{DPP}\rangle,|\mathrm{DPP}, \mathrm{DPP}\rangle$ involving the UP state are also shown. (c)-(f) Liouville path analysis of nonlinear interactions. (c) Decomposition of the four-wave-mixing processes of the LP (blue arrows), DPP (red arrows), and UP resonances (green arrows), individually. (d) Liouville path for a four-wave-mixing process combining the nonlinear polarization of the LP and the DPP. (e),(f) Fourwave-mixing processes mixing the UP and DPP resonances. The black dot shows the virtual level $|\mathrm{UP}, \mathrm{DPP}\rangle$ [see (b)]. 
(4WM) signals along the red dashed lines depend on the phases of both fields and probe the nonlinear polarization, $P_{4 \mathrm{WM}}^{(3)}\left(\nu_{j}\right)=\chi^{(3)}\left(\nu_{j}, \nu_{j},-\nu_{j}\right) \mathcal{A}_{m}\left(\nu_{j}\right) \mathcal{A}_{m}\left(\nu_{j}\right) \mathcal{A}_{n}\left(-\nu_{j}\right)$. We illustrate this wave vector decomposition [Fig. 3(b)] for the 4WM signals located at $\left(\nu_{j},-\nu_{j}\right)$ in Fig. 3(c), where blue, red, and green arrows mark the Liouville paths for the LP, DPP, and UP resonances, respectively. In each case, $P_{4 \mathrm{WM}}^{(3)}\left(\nu_{j}\right)$ requires absorbing two photons from $\mathcal{E}_{\mathrm{A}}$ (horizontal arrows) and emitting one photon into $\mathcal{E}_{\mathrm{B}}$ (diagonal arrows), creating a polarization at a frequency of $\nu_{j}$ whose emission is subsequently detected electro-optically. All photons are resonant to the respective polariton. Equivalent signatures, yet with up to eight-wave-mixing processes, are obtained for a sample containing a single QW [26].

Intriguingly, $\mathcal{A}_{\mathrm{nl}}$ reveals additional maxima at frequencies such as $\left(\nu_{\mathrm{LP}},-\nu_{\mathrm{DPP}}\right)$ resulting from nonlinear polarization mixing between different polariton resonances [Fig. 3(d)]. Here, $\mathcal{E}_{\mathrm{A}}$ contributes a photon resonant to the LP (blue arrow) and one resonant to the DPP (red horizontal arrow), while a photon resonant to the DPP is emitted into $\mathcal{E}_{\mathrm{B}}$ (red diagonal arrow), driving a nonlinear polarization $P_{4 \mathrm{WM}}^{(3)}\left(\nu_{\mathrm{LP}}\right)=$ $\chi^{(3)}\left(\nu_{\mathrm{LP}}, \nu_{\mathrm{DPP}},-\nu_{\mathrm{DPP}}\right) \mathcal{A}_{\mathrm{A}}\left(\nu_{\mathrm{LP}}\right) \mathcal{A}_{\mathrm{A}}\left(\nu_{\mathrm{DPP}}\right) \mathcal{A}_{\mathrm{B}}\left(-\nu_{\mathrm{DPP}}\right)$. Equivalent processes mixing the polarizations of the UP and the DPP are highlighted in Figs. 3(e) and 3(f), respectively. These interpolariton signatures mark a strong-field limit of ultrastrong coupling in which the normal-mode approximation is violated. The broad variety of nonlinear interactions resulting from all possible wave vector combinations [26] underscores the high design flexibility regarding custom nonlinearities of light-matter hybrid states by subwavelength design of ultrastrong coupling.

The observation of nonlinear interactions of independent normal modes calls for a quantitative theoretical description implementing the coupling of the resonator fields to the electronic polarization of the multilevel Landau fan. In second quantization, our Hamiltonian,

$$
\begin{aligned}
\hat{H}= & \sum_{j} \hbar \omega_{j} \hat{a}_{j}^{\dagger} \hat{a}_{j}+\hbar \omega_{c} \hat{b}^{\dagger} \hat{b} \\
& +\hbar \sum_{j} \Omega_{R, j}^{v}\left(\hat{a}_{j}+\hat{a}_{j}^{\dagger}\right)\left(\hat{b}+\hat{b}^{\dagger}\right) \\
& +\hbar \sum_{j} D_{j}\left(\hat{a}_{j}+\hat{a}_{j}^{\dagger}\right)^{2}+\hat{H}_{\mathrm{ext}},
\end{aligned}
$$

contains bosonic annihilation operators $\hat{a}_{j}$ and frequencies $\omega_{j}$ for the $j$ th cavity mode and their cyclotron electronic counterparts $\hat{b}$ and $\omega_{c}$. The respective bare energies motivate the first two terms. The third term couples the cavity modes to the electronic polarization by vacuum Rabi frequencies $\Omega_{R, j}^{v}$ including antiresonant interactions $\hat{a}_{j} \hat{b}+$ H.c. The fourth term treats the diamagnetic shift of the cavity modes, $D_{j}=\Omega_{R, j}^{v}{ }^{2} / \omega_{c}$. Finally, $\hat{H}_{\text {ext }}$ couples the cavity to the far field.

We obtain the polarization dynamics by Heisenberg's equation of motion, in a mean-field limit. A comparison with the experimental data allows for calibrating $\Omega_{R, j}^{v}$, damping terms, and the Rabi frequency of the external field, $\Omega_{R}=2 \pi \times 2.7 \mathrm{THz}$ [26]. While this bosonic description is valid for weak fluences, strong-field excitation unravels the fermionic nature of the electronic system which we implement by a density matrix formalism that considers the coherent polarization and incoherent populations of $10^{2}$ Landau levels (LLs). The density matrix $\rho$ contains the LL populations $n_{l}=\rho_{l l}$ on the diagonal and the inter-LL polarizations in the adjacent minor diagonal, $p_{l}=\rho_{l, l+1}=\rho_{l+1, l}^{*}$. The total polarization $\beta=\sum_{l} p_{l}$ is coupled back to the cavity. The dynamics of $\rho$ including damping and relaxation is obtained via the von Neumann equation [26]. Figure 4(a) shows the evolution of the population of each LL for the situation of Fig. 2(c). Within the FWHM of $\mathcal{E}_{\mathrm{B}}$ of $2 \mathrm{ps}$ around $t=0 \mathrm{ps}$, Landau electrons are coherently excited from up to 5 rungs below to 5 rungs above the Fermi level. The maximum energy spread remains slightly below the energy of the longitudinal optical phonon of GaAs. This threshold is exceeded by doubling $\mathcal{E}_{\mathrm{B}}$, leading to rapid electron-phonon scattering and strong dephasing [26]. We condense the complex system state into an excitation parameter $\rho_{\text {exc }}$, which quantifies the number of Landau excitations relative to thermal equilibrium, and corresponds to $|\beta|^{2}$ of the bosonic Hamiltonian, without nonlinearities [26]. After excitation, the periodic energy exchange of the cavity and the Landau electrons causes periodically alternating local maxima of $\rho_{\text {exc }}$ and $\left|\alpha_{j}\right|$ [Figs. 4(b) and 4(c)].

Moreover, our model explains the microscopic origin of the nonlinearities. A first potential mechanism is given by a nonequidistant progression of LL energies, as was shown for Dirac electrons in graphene [34]. Including the nonparabolic band structure of GaAs and its slightly nonlinear spacing of LL frequencies [26,35], the calculated dynamics qualitatively match the experiment, yet evolve much slower [Figs. 4(d) and 4(e)] and exhibit a redshifted spectrum dominated by the LP resonance. Moreover, the peak amplitude of $\mathcal{A}_{\mathrm{nl}}$ reaches only $8 \%$ of the experimentally observed value, while surpassing the noise floor of $1.2 \%$ of the experimental peak amplitude [26]. This evidences the presence of additional, strong nonlinear contributions.

While gold resonators are expected to respond linearly up to much stronger fields [36], the CR has been shown to exhibit nonlinearities beyond Kohn's theorem by dynamical Coulomb correlations at field amplitudes of a few $\mathrm{kV} / \mathrm{cm}$ [32]. To first order, the resulting renormalization of the LL energies and the inter-LL dipole moments depends on the instantaneous degree of excitation $\rho_{\text {exc }}(t)$. Scaling both effects for a quantitative fit with the experiment [26], 
(a)

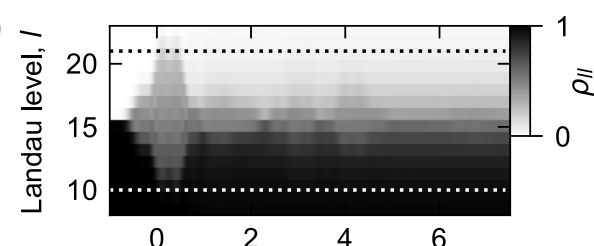

(b)

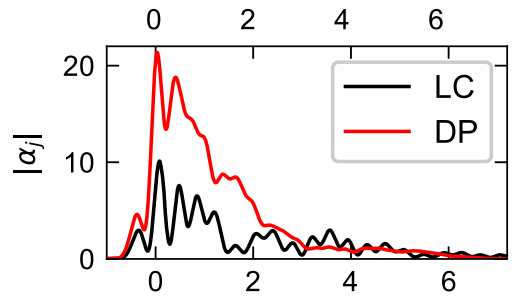

(c)

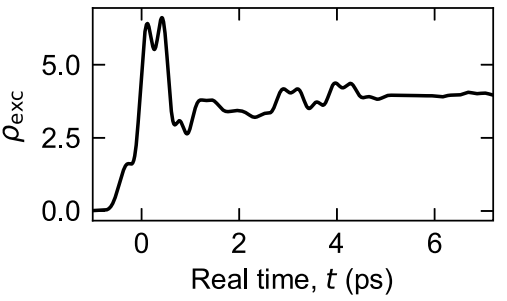

(d)

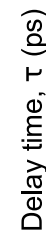

(f)

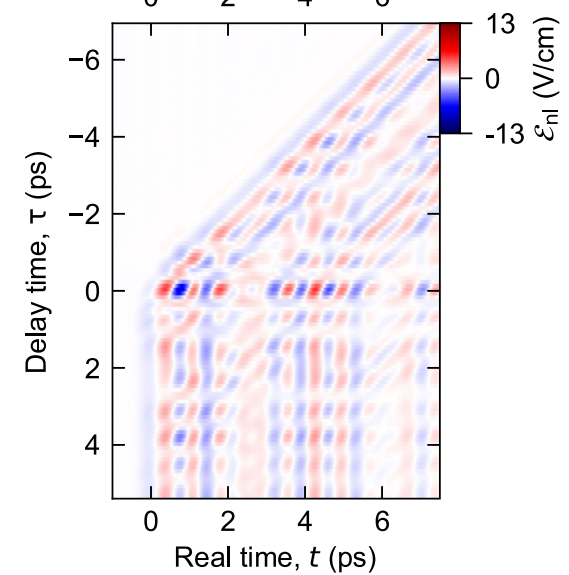

(e)

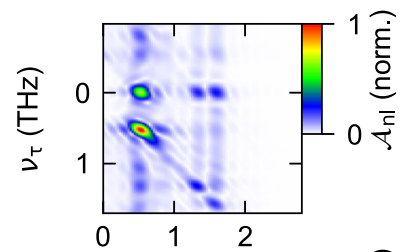

(g)

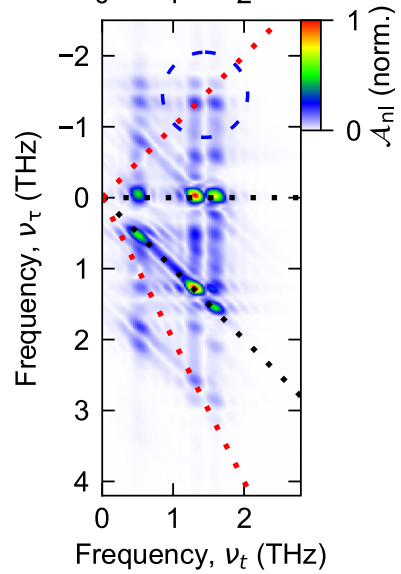

FIG. 4. Calculation results. (a) Population of each Landau level for the setting of Fig. 2(c). The dashed lines show the threshold energy for longitudinal optical phonon scattering (see text). (b) Population of the LC and DP cavity modes for $\tau=0$ ps. (c) Electronic excitation $\rho_{\text {exc }}$. (d) Calculated response $\mathcal{E}_{\mathrm{nl}}(t, \tau)$ considering only band structure effects. (e) Amplitude spectrum of the data of (d), normalized to its peak value. (f) $\mathcal{E}_{\mathrm{nl}}(t, \tau)$ from our full theory including Coulomb effects. (g) Amplitude spectrum of the data of (f), normalized to its peak value.

the full calculation [Fig. 4(f)] reproduces the rapid oscillations originating from the UP and DPP resonances, and the slower modulation induced by the LP resonance. Moreover, relative and absolute spectral amplitudes of pump-probe and four-wave-mixing signals are adequately rendered for the individual polaritons, and for the nonlinear correlations between separate polariton resonances [Fig. 4(g)]. A switch-off analysis shows that only the combination of all nonlinearities enables this quantitative agreement [26]. Our theory describes the creation of distinct resonances by strong-field interactions of lightmatter hybrid modes which greatly expands the parameter space for tailoring deep-strong coupling - a regime that was hitherto restricted to linear interactions with weak probe fields.

In conclusion, we have investigated a novel regime of ultrastrong light-matter coupling where subcycle polarization dynamics of a many-body electronic system are driven by both vacuum fluctuations and a coherent external field. Nonperturbative excitation stimulates pump-probe and coherent multiwave mixing nonlinearities. Moreover, nonlinearities linking the polarization of separate polaritons break the normal-mode approximation dynamically, creating resonances inaccessible by linear spectroscopy. Whereas deep-strong coupling features light-matter hybrid modes with exotic properties in equilibrium, the nonlinear interactions of eigenstates observed here open up previously inaccessible degrees of freedom of cavity quantum electrodynamics. This strategy may unveil quantum phenomena in similarity to cavity quantum electro- dynamics in the strong-coupling regime, where BoseEinstein condensates or quantum fluids of light have been routinely created [37]. Following a similar philosophy, nonlinear optical control of deep-strong coupling could massively enhance ground-state instabilities and phase transitions in superconductivity [21,22], cavity chemistry $[19,20]$, or vacuum-modified electronic transport [14,18], and enable the detection of quantum vacuum radiation [38]. The comparably low field amplitudes on the order of $1 \mathrm{kV} / \mathrm{cm}$ may facilitate innovative quantum devices exploiting parametric polariton nonlinearities or the generation of squeezed quantum states of light.

We thank Andreas Bayer for experimental support and gratefully acknowledge support by the DFG through Grants No. LA 3307/1-2, No. BO 3140/3-2, and No. HU 1598/8, as well as by the European Research Council (ERC) through Future and Emerging Technologies (FET) Grant No. 737017 (MIR-BOSE).

*rupert.huber@ur.de †christoph.lange@tu-dortmund.de

[1] A. H. Chin, O. G. Calderón, and J. Kono, Phys. Rev. Lett. 86, 3292 (2001).

[2] B. Zaks, R. B. Liu, and M. S. Sherwin, Nature (London) 483, 580 (2012).

[3] O. Schubert et al., Nat. Photonics 8, 119 (2014).

[4] F. Langer et al., Nature (London) 533, 225 (2016).

[5] O. D. Mücke, T. Tritschler, M. Wegener, U. Morgner, and F. X. Kärtner, Phys. Rev. Lett. 87, 057401 (2001). 
[6] J. Raab et al., Opt. Express 27, 2248 (2019).

[7] G. Günter et al., Nature (London) 458, 178 (2009).

[8] A. A. Anappara, S. De Liberato, A. Tredicucci, C. Ciuti, G. Biasiol, L. Sorba, and F. Beltram, Phys. Rev. B 79, 201303 (R) (2009).

[9] Y. Todorov, A. M. Andrews, R. Colombelli, S. De Liberato, C. Ciuti, P. Klang, G. Strasser, and C. Sirtori, Phys. Rev. Lett. 105, 196402 (2010).

[10] G. Scalari et al., Science 335, 1323 (2012).

[11] J. Keller, G. Scalari, S. Cibella, C. Maissen, F. Appugliese, E. Giovine, R. Leoni, M. Beck, and J. Faist, Nano Lett. 17, 7410 (2017).

[12] A. Bayer, M. Pozimski, S. Schambeck, D. Schuh, R. Huber, D. Bougeard, and C. Lange, Nano Lett. 17, 6340 (2017).

[13] X. Li, M. Bamba, Q. Zhang, S. Fallahi, G. C. Gardner, W. Gao, M. Lou, K. Yoshioka, M. J. Manfra, and J. Kono, Nat. Photonics 12, 324 (2018).

[14] G. L. Paravicini-Bagliani et al., Nat. Phys. 15, 186 (2019).

[15] A. F. Kockum, A. Miranowicz, S. De Liberato, S. Savasta, and F. Nori, Nat. Rev. Phys. 1, 19 (2019).

[16] P. Forn-Díaz, L. Lamata, E. Rico, J. Kono, and E. Solano, Rev. Mod. Phys. 91, 025005 (2019).

[17] F. Yoshihara, T. Fuse, S. Ashhab, K. Kakuyanagi, S. Saito, and K. Semba, Nat. Phys. 13, 44 (2017).

[18] E. Orgiu et al., Nat. Mater. 14, 1123 (2015).

[19] R. Chikkaraddy, B. de Nijs, F. Benz, S. J. Barrow, O. A. Scherman, E. Rosta, A. Demetriadou, P. Fox, O. Hess, and J. J. Baumberg, Nature (London) 535, 127 (2016).

[20] A. Thomas et al., Science 363, 615 (2019).

[21] F. Schlawin, A. Cavalleri, and D. Jaksch, Phys. Rev. Lett. 122, 133602 (2019).

[22] A. Thomas et al., arXiv:1911.01459.

[23] A. Ridolfo, M. Leib, S. Savasta, and M. J. Hartmann, Phys. Rev. Lett. 109, 193602 (2012).
[24] A. Ridolfo, S. Savasta, and M. J. Hartmann, Phys. Rev. Lett. 110, 163601 (2013).

[25] A. F. Kockum, A. Miranowicz, V. Macrì, S. Savasta, and F. Nori, Phys. Rev. A 95, 063849 (2017).

[26] See Supplemental Material at http://link.aps.org/ supplemental/10.1103/PhysRevLett.126.177404, which contains an in-depth discussion of the theory, more details on the experimental setup, additional data, and Refs. [27,28].

[27] K. von Klitzing, Rev. Mod. Phys. 58, 519 (1986).

[28] C. Ciuti, G. Bastard, and I. Carusotto, Phys. Rev. B 72, 115303 (2005).

[29] W. Kuehn, K. Reimann, M. Woerner, and T. Elsaesser, J. Chem. Phys. 130, 164503 (2009).

[30] W. Kuehn, K. Reimann, M. Woerner, T. Elsaesser, R. Hey, and U. Schade, Phys. Rev. Lett. 107, 067401 (2011).

[31] F. Junginger, B. Mayer, C. Schmidt, O. Schubert, S. Mährlein, A. Leitenstorfer, R. Huber, and A. Pashkin, Phys. Rev. Lett. 109, 147403 (2012).

[32] T. Maag et al., Nat. Phys. 12, 119 (2016).

[33] A. G. Stepanov, J. Hebling, and J. Kuhl, Appl. Phys. Lett. 83, 3000 (2003).

[34] J. C. König-Otto, Y. Wang, A. Belyanin, C. Berger, W. A. de Heer, M. Orlita, A. Pashkin, H. Schneider, M. Helm, and S. Winnerl, Nano Lett. 17, 2184 (2017).

[35] W. Zawadzki, C. Chaubet, D. Dur, W. Knap, and A. Raymond, Semicond. Sci. Technol. 9, 320 (1994).

[36] C. Lange, T. Maag, M. Hohenleutner, S. Baierl, O. Schubert, E. R. J. Edwards, D. Bougeard, G. Woltersdorf, and R. Huber, Phys. Rev. Lett. 113, 227401 (2014).

[37] I. Carusotto and C. Ciuti, Rev. Mod. Phys. 85, 299 (2013).

[38] S. De Liberato, C. Ciuti, and I. Carusotto, Phys. Rev. Lett. 98, 103602 (2007). 